\title{
Des Fata aux fées : regards croisés de l'Antiquité à nos jours
}

\author{
Martine Hennard Dutheil de la Rochère et Véronique Dasen
}

\section{(2) OpenEdition}

1 Journals

Édition électronique

URL : http://journals.openedition.org/edl/136

DOI : $10.4000 /$ edl. 136

ISSN : 2296-5084

Éditeur

Université de Lausanne

\section{Édition imprimée}

Date de publication : 15 décembre 2011

Pagination : 15-34

ISBN : 978-2-940331-26-0

ISSN : 0014-2026

\section{Référence électronique}

Martine Hennard Dutheil de la Rochère et Véronique Dasen, « Des Fata aux fées : regards croisés de I'Antiquité à nos jours », Études de lettres [En ligne], 3-4 | 2011, mis en ligne le 15 décembre 2014, consulté le 18 décembre 2020. URL : http://journals.openedition.org/edl/136 ; DOI : https://doi.org/ 10.4000/edl. 136

\section{(C) Études de lettres}




\section{DES FATA AUX FÉES : \\ REGARDS CROISÉS DE L'ANTIQUITÉ À NOS JOURS*}

Dans une célèbre tirade de Romeo and Juliet, Mercutio s'emporte en accusant Roméo amoureux d'avoir été visité nuitamment par la Reine Mab, «the fairies' midwife» («la sage-femme des fées» mais aussi «l'accoucheuse des rêves"), dans une description fantastique qui influencera durablement l'imaginaire féerique. L'ami de Roméo décrit la Reine Mab comme un personnage minuscule perché sur le nez des dormeurs, qui a la capacité de traduire en rêve leurs vœux, espoirs, craintes ou désirs les plus secrets. Prenant une tournure érotique et inquiétante au gré de l'imagination de Mercutio, la figure se métamorphose en une vieille femme pressant les jeunes filles endormies dans une image qui mêle les angoisses du cauchemar (le succube), l'étreinte amoureuse et les douleurs de l'enfantement:

This is the hag, when maids lie on their back,

That presses them and learns them first to bear,

Making them women of good carriage (acte I, scène IV, vers 92-94).

Marina Warner en déduit que la féerie ne met pas seulement en lumière l'expérience intime et les ressorts inconscients de la psyché humaine, «bien que ces histoires tirent leur séduction de leurs enchantements et de leur fantaisie»: "elles traitent aussi des expériences et des savoirs de communautés humaines qui ont la plupart du temps été ignorées ou passées sous silence: les femmes, les enfants, les pauvres» ${ }^{1}$.

* Les éditrices souhaitent remercier les responsables de la CUSO et de la Faculté des lettres pour le financement du colloque, la Société Académique Vaudoise, Brigitte Maire pour ses conseils avisés, Marie-Emilie Walz et Olivier Knechciak pour leur 
Les figures féminines sont plurielles: concentrant une partie des "expériences et des savoirs» dont parle Warner, elles sont des déessesmères, des divinités tutélaires, des sages-femmes, jusqu'à former un cortège de fées bienveillantes ou menaçantes, qui deviendront souvent les déléguées de l'auteur(e) dans la tradition littéraire. Les liens qui les unissent sont bien connus ${ }^{2}$. On sait que le mot fée (fata en italien, hada en espagnol, fairy en anglais), est issu de l'ancien français faie ou fee, désignant les personnages légendaires issus du folklore et des romans médiévaux. Le terme lui-même provient du latin Fata, divinités féminines personnifiant le destin. Fata est lié étymologiquement à fatum, participe passé du verbe fari (de la racine indo-européenne *bhã-), qui signifie parler, et par extension "chose dite, décision, décret", "déclaration prophétique, prédiction», et donc "destin» (fate en anglais). Comme les Moĩpal grecques déterminant la "part" (moira) de vie revenant à chacun, les Parques (Clotho qui file, Lachésis qui dévide et Atropos qui coupe le fil de la vie), Fata et fées allient à leur fonction tutélaire la force d'une parole créatrice et performative ${ }^{3}$. Les Fata sont donc reliées à la fois au destin et à la naissance.

aide précieuse et leur relecture attentive de plusieurs articles, ainsi que les rédactrices d'Etudes de Lettres, Florence Bertholet et Catherine Chène pour leur professionalisme. Nos vifs remerciements vont aussi aux deux lectrices externes, Michelle RyanSautour et Jacqueline Fabre-Serris, pour avoir accepté de rejoindre le comité éditorial du volume. Neil Forsyth et Irène Kacandes nous ont également fait l'amitié de relire quelques articles du volume. Beaucoup de fées marraines se sont penchées sur le berceau de ce volumineux bébé.

I. "The stories are not only fantastical, though their appeal arises from their enchantments and fancifulness; they also encode a great deal of experience and knowledge from among the usually unnoticed and the voiceless groups: women, children, the poor» (M. Warner, "After Rapunzel», p. 329). Notre traduction. Voir aussi l'ouvrage de référence de M. Warner, From the Beast to the Blonde.

2. Les mythographes et folkloristes du XIX ${ }^{\mathrm{e}}$ siècle, dont les frères Grimm, ont documenté ces origines antiques. Voir aussi P. C. van der Horst, «Fatum, Tria Fata; Parca, Tres Parcae». Plus récemment, Graham Anderson a enquêté sur les sources antiques des contes dans Fairytale in the Ancient World. On lira avec profit l'ouvrage de S. BallestraPuech, Les Parques, sur la réception littéraire des figures antiques du destin. Sur l'héritage antique des romans médiévaux, voir E. Faral, Recherches sur les sources latines des contes et romans courtois du Moyen Age.

3. Voir S. Ballestra-Puech, Les Parques. Le nom des Carmentes romaines est d'ailleurs dérivé de carmen, le chant incantatoire ou la formule magique; cf. l'article de V. Dasen dans ce volume. 
Le présent volume réunit des contributions qui visent à éclairer dans une perspective historique, anthropologique, culturelle et littéraire la mise en œuvre de l'imaginaire de la naissance et de la destinée humaine dont la scène inaugurale du conte de la Belle au bois dormant sert de point de référence. Il s'attache aux rites, cultes, croyances, récits et représentations entourant ce moment décisif où les femmes jouent un rôle prépondérant, depuis la plus haute Antiquité jusqu’aux versions modernes et contemporaines du conte familier.

Des Fata aux fées: regards croisés de l'Antiquité à nos jours est issu du colloque interdisciplinaire qui a eu lieu les 7 et 8 octobre 2009 à l'Université de Lausanne, organisé par Martine Hennard Dutheil de la Rochère et Véronique Dasen. Ce colloque international a permis à des spécialistes issus de différentes disciplines de se pencher sur une thématique commune, centrée sur les figures des Moires, des Parques et de leurs avatars modernes, les fées. Inscrits depuis l'Antiquité dans l'imaginaire collectif, ces personnages féminins ont fait l'objet de constantes réinterprétations dans diverses cultures et à différentes époques jusqu'à désigner un genre littéraire, le conte de fées, qui connaîtra le succès et le destin international que l'on sait.

La gravure de Sleeping Beauty par Walter Crane choisie pour la couverture du volume illustre notre démarche, qui vise à créer les conditions d'un dialogue entre des spécialistes des textes et des images, des littératures et des cultures anciennes, médiévales, modernes et contemporaines autour de La Belle au bois dormant ${ }^{4}$. Le conte de Perrault, souvent confondu avec la version plus tardive des Grimm, constitue le fil rouge du volume, car cette "histoire du temps passé» nous éclaire sur la façon dont les figures du destin et les rites de naissance qui lui sont associés ont été réinterprétés au fil du temps.

Crane déplace notre attention du baiser romantique introduit par les Grimm sur la scène inaugurale qui permet de saisir l'épaisseur historique de cette vieille histoire: les figures du destin qui se penchent sur le berceau de la petite princesse ont en effet une origine très lointaine et une histoire complexe. Elles ont fait l'objet de reprises, variations et transformations successives qui, chacune, $s^{\prime}$ inscrit dans un contexte culturel spécifique, souvent en écho, réponse ou réaction à d'autres représentations passées ou présentes du même thème. En réunissant l'incipit du

4. W. Crane, The Bluebeard Picture Book, 1875. 


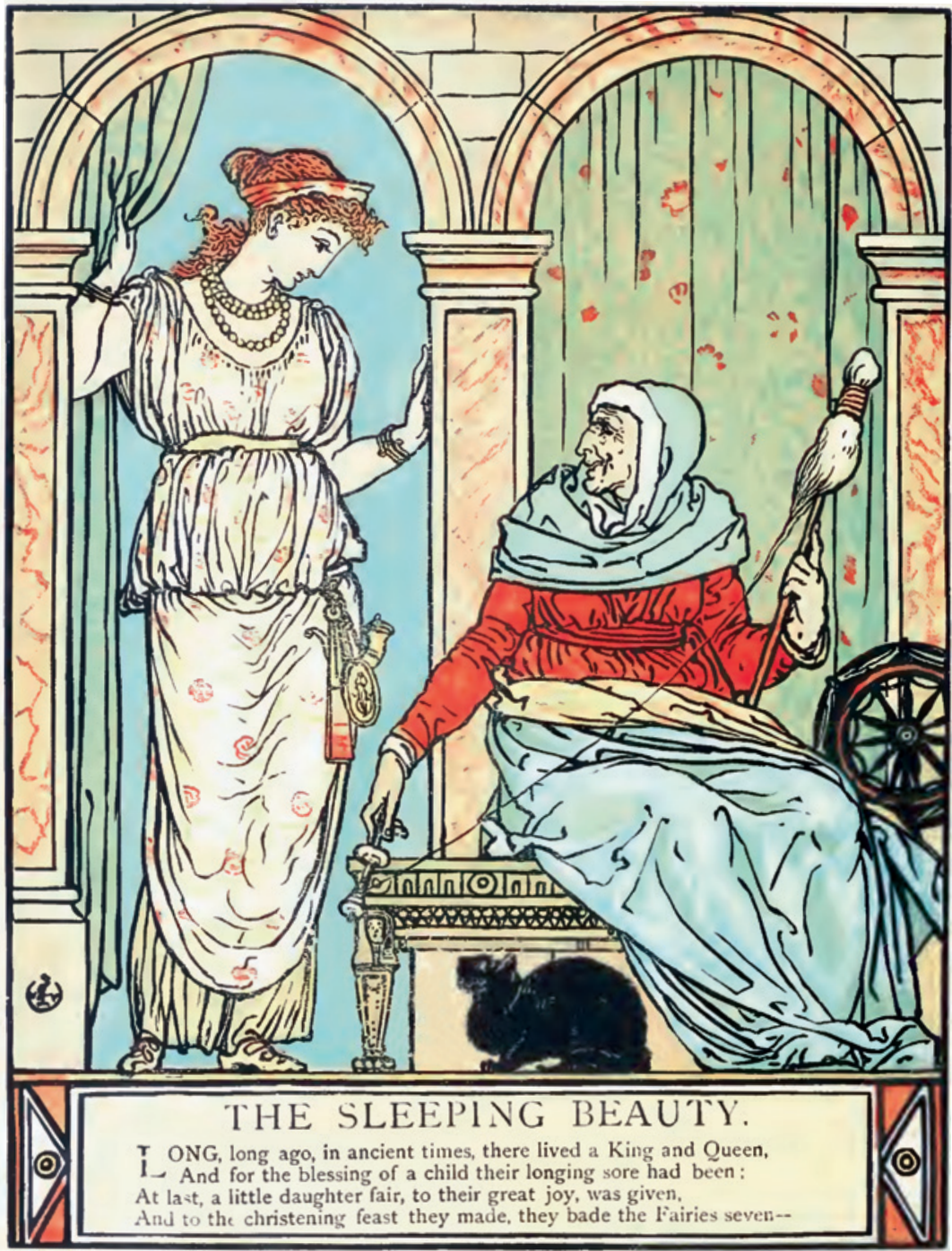


conte et la scène du fuseau fatal à la jeune fille, l'illustration de Crane amalgame deux moments distincts de l'intrigue: la naissance placée sous le signe des fées et la réalisation de la malédiction. Elle nous rappelle par cette ellipse temporelle que la question du temps est au cœur de La Belle au bois dormant, comme d'ailleurs du genre du "conte du temps passé» dans son ensemble, dont la nature même est d'être raconté, et donc constamment recontextualisé, réactualisé et réinventé. Elle réunit aussi le conte "pour enfants» et l'illustration, qui connaissent un succès sans précédent en cette fin du XIX siècle $^{5}$. Emblématique du mouvement Arts and $\mathrm{Crafts}^{6}$, l'illustration de Crane mêle les citations stylistiques aux enluminures médiévales et à l'architecture néo-antique qu'illustrent aussi les vêtements et la posture de la Belle. Crane rappelle ainsi que le conte récapitule des périodes et des héritages divers: il puise dans de très anciennes représentations du destin symbolisées ici par la vieille fileuse qui évoque à la fois les Parques antiques et la sorcière Carabosse accompagnée de son chat noir, la parole prophétique et les contes de vieille associés au rouet. L'image intègre aussi la tradition écrite avec quelques extraits du conte de Perrault traduits et mis en vers au bas de l'image: le récit est situé dans un passé lointain («in ancient times»), conformément aux conventions du genre, même si la prédiction des fées projette aussitôt brutalement l'histoire dans l'avenir: la joie de la naissance tant attendue du bébé ("a little daughter fair, to their joy was given ») fait rimer le don de l'enfant avec les dons bien plus ambigus que les «Fairies seven» font à la jeune princesse condamnée à un sombre destin. Crane rassemble ainsi dans cette image les fils tissés dans le volume, qui cherche à rendre sensible l'épaisseur historique et la dimension palimpseste du conte en croisant les époques et les cultures, les arts et les lettres, de façon à mieux saisir les liens qui unissent les figures féminines du destin et les fées modernes, de l'Antiquité à l'époque contemporaine.

5. Sur la vogue de l'illustration au XIX ${ }^{\mathrm{e}}$ siècle, cf. l'article de Ph. Kaenel.

6. Walter Crane (1845-1915) est un peintre, dessinateur et illustrateur anglais célèbre pour ses illustrations de livres pour enfants réalisées dans un style archaïsant. Avec William Morris, il fonda le mouvement Arts and Crafts dans le but de réformer les arts décoratifs en intégrant l'art à tous les aspects de la vie quotidienne. Crane réalisa des papiers peints (notamment inspirés de Sleeping Beauty) et illustra de nombreux livres de contes, dont The Frog Prince (1874) et Household Stories from Grimm (1882), ainsi que The Faerie Queene d'Edmund Spenser (1894-1896). 
Situé au croisement de plusieurs disciplines, ce volume considère le conte comme un objet complexe et protéiforme qui requiert une mise en contexte historique, sociale et culturelle, et un éclairage interdisciplinaire. Comme le souligne Jack Zipes, le conte n'existe pas $^{7}$ : parce qu'ils voyagent et se transmettent sous des formes diverses, les contes se transforment selon les époques et les lieux, les sociétés et les cultures, les langues et les supports, mais aussi les visées de celles et ceux qui les racontent. La nature dynamique et dialogique du genre appelle ce type de démarches transverses, comme le proposait déjà Donald Haase dans sa préface à Fairy Tales and Feminism: New Approaches (2004), qui nous a fait l'honneur et l'amitié de présenter la conférence plénière. Sa contribution montre comment se rejoue la relation entre oralité et écriture dans quelques versions clés du conte qui témoignent de sa double origine. Cette question est d'autant plus importante qu'elle soulève une problématique centrale dans les études sur le conte, qui voient s'affronter les approches issues de la folkloristique d'une part, et les études philologiques et sociohistoriques d'autre part, les unes privilégiant la parole, l'oralité et les traditions populaires, les autres l'écrit, la tradition littéraire, et les phénomènes de transmission et de réception textuelle. A l'image de "Kiss and Tell», le volume vise à mettre en dialogue ces types d'analyse complémentaires qui envisagent le conte comme un fait de culture lié à la parole, à l'image et aux productions culturelles prises au sens large, mais s'attachent aussi à retrouver leur trace écrite dans le détail des textes et l'histoire du livre ${ }^{8}$. Offrant une perspective transversale et diachronique, le volume croise ainsi les savoirs et les approches dans le but de renouveler notre compréhension du conte au-delà des clivages théoriques ou méthodologiques. Sa longue histoire se lit ici dans les traces archéologiques, la sculpture funéraire, le manuscrit et l'imprimé, mais aussi l'illustration, le dessin animé, la pièce radiophonique, la photographie, la bande dessinée et le livre pour enfants, entre autres. Le volume nous fait ainsi entrer dans la «fabrique du conte», pour citer Sylvie BallestraPuech, des récits mésopotamiens jusqu’au cinéma contemporain.

7. "There is no such thing as the fairy tale; however, there are hundreds of thousands of fairy tales" (J. Zipes (éd.), The Oxford Companion to the Fairy Tale, p. Xv). Outre les travaux de J. Zipes, voir aussi l'ouvrage fondateur de C. Velay-Vallantin, L'histoire des contes.

8. En témoigne la récente controverse provoquée par l'ouvrage de l'historienne du conte R. B. Bottigheimer, Fairy Tales: A New History. 
Le volume est composé de trois parties: la première, intitulée "Autour des Moires et des Parques: cultes, rites, représentations", rassemble des contributions relatives aux plus anciennes représentations des rites de naissance qui ont lointainement inspiré le conte familier. La deuxième partie, "Des déesses antiques aux fées modernes: réécritures littéraires", porte sur la tradition textuelle et littéraire du conte de La Belle au bois dormant, des sources latines jusqu'aux réécritures modernes. La troisième partie, "Transmission culturelle: paroles, textes, images», s'attache plus largement à sa transmission culturelle à travers l'illustration, la littérature pour la jeunesse, la photographie, le dessin animé, la radio ou encore le cinéma.

Autour des Moires et des Parques: cultes, rites, représentations

Cette première partie rassemble quatre articles qui révèlent à la fois la cohérence et la diversité des rites et des représentations autour de la naissance, de la Mésopotamie ancienne à l'époque romaine impériale, en passant par l'Egypte et la Grèce. Dans chacune de ces cultures antiques, les figures divines qui président au destin du nouveau-né sont principalement féminines; elles détiennent une parole à valeur prophétique qui s'exerce dès le premier cri, au moment de couper le cordon ombilical ou lors du premier bain. Toutes mettent en œuvre le pouvoir caché des femmes, divines ou humaines, à l'aube de la vie. Leurs compétences sont amplifiées par leur démultiplication, et leur ambivalence se traduit par leur patronage des deux extrêmes du cycle de la vie, la naissance et la mort.

En Mésopotamie, la déesse-mère qui préside au façonnage de l'enfant dans le ventre maternel fixe ainsi sa destinée en prononçant «le bon mot» au moment de la section du cordon ombilical. Il s'agit peut-être d'une formule indiquant son "chemin de vie» inscrit sur la "tablette des destinées» gardée par Enlil, le père des dieux. Constance Frank, dans " "Le fuseau et la quenouille”. Personnalités divines et humaines participant à la naissance de l'homme et à sa destinée en Mésopotamie ancienne", documente les textes mythologiques, les formules incantatoires et l'iconographie qui se rapportent à ce rite de passage. On notera les intrications entre le monde des vivants et des morts: l'enfant in utero se forme dans des eaux souterraines et quitte métaphoriquement «le quai de la 
mort " au moment de la naissance qui le mènera à l'accomplissement de son destin, c'est-à-dire le retour à la mort. Les divinités essentiellement féminines qui président à la naissance peuvent être redoutables. La démone Lamaštu, créature hybride, personnifie les menaces qui rôdent la nuit. Elle tue, dit-on, les femmes en couche et les bébés en se substituant à la nourrice, comme le feront plus tard aussi les effrayantes Striges des Romains qui sont ses lointaines héritières?

Cette ambivalence se retrouve dans le monde égyptien où la naissance se déroule en musique, comme les rites funéraires destinés à assurer la renaissance du défunt. Cathie Spieser, dans "Meskhenet et les Sept Hathors en Egypte ancienne», se base principalement sur des récits dont la nature peut être rapprochée de celle des "contes" modernes, mêlant figures humaines et surnaturelles, œuvres de lettrés, objets de réécritures régulières, mais destinés à une lecture orale pour un large auditoire. Comme en Mésopotamie, le destin est fixé verbalement au moment de la naissance. Dans le Papyrus Westcar, Isis annonce le destin du futur roi en déclarant son nom, tandis que Meskhenet intervient lors du premier bain avec une parole prophétique. La pluralité des quatre Meskhenet et des sept Hathors offre un parallèle au redoublement de figures similaires $\mathrm{du}$ monde grec et romain. Le motif du fil ou cordon se dessine dans les sources égyptiennes: les déesses sont associées à des bandelettes rouges qui pourraient se rapporter au cordon ombilical, un attribut qui est promesse de renaissance du mort quand le cordon rouge est porté par le dieu chacal Anubis.

Dans le monde grec, la multiplicité des divinités qui président aux naissances prend la forme des Moires, chez Hésiode au nombre de trois, dont l'identité et la fonction sont minutieusement analysées par Vinciane Pirenne-Delforge et Gabriella Pironti, dans «Les Moires entre la naissance et la mort: de la représentation au culte». Si les Moires, comme ailleurs en Méditerranée, président à la fois à la naissance et à la mort de l'individu, elles se distinguent en accordant plus spécifiquement la "part», moira, qui revient à chacun. Puissances de distribution, de rétribution et de régulation, les Moires sont solidaires de l'équilibre garanti par l'autorité souveraine de Zeus et contribuent à le gérer au sein des communautés humaines. Avec les Moires apparaît au moment de l'accouchement

9. Sur la généalogie que l’on peut retracer de Lamaštu aux Striges, voir par exemple I. Sorlin, «Striges et Geloudes». 
la figure traditionnelle de la fileuse, puis de la tisserande, associée à la métaphore de la trame de la vie et des dessins variés qui la constituent.

Dans chaque culture, les divinités féminines se superposent aux auxiliaires humaines de la naissance dont la silhouette se dessine plus ou moins clairement dans les sources. L'iconographie des sarcophages romains offrent de nombreuses scènes du premier bain, où, comme les Meskhenet égyptiennes, les Parques fixent le destin de l'enfant. Un ensemble de gemmes méconnues livrent une nouvelle clé d'interprétation du moment de la section du cordon ombilical. Véronique Dasen, dans «Le pouvoir des femmes: des Parques aux Matres», démontre la valeur rituelle de ce geste que la sage-femme accomplit sous les regards des trois Parques. Pour le ligaturer, elle utilise un cordon de laine semblable à celui que filent ses doubles surnaturels. Un premier cycle est donc bouclé quand on procède au premier bain qui intègre l'enfant dans le monde des vivants. Loin d'être soumises à la toute-puissance d'un pater familias, ce sont les femmes, humaines et divines, qui président à l'entrée dans la vie de l'enfant.

\section{Des déesses antiques aux fées modernes: réécritures littéraires}

Cette deuxième partie opère le lien entre l'héritage gréco-romain et les contes de fées modernes. On sait que l'époque médiévale a accordé une place importante au merveilleux, puisé dans des sources mythiques, littéraires, populaires et religieuses. De fait, les relations entre merveilleux profane et chrétien sont particulièrement complexes ${ }^{10}$. La

Io. Sur les fées dans la littérature du Moyen Age, voir L. Harf-Lancner, Les fées au Moyen Age: Morgane et Mélusine, suivi de Les mondes des fées dans l'occident médiéval. Comme le rappelle B. Ribémont, Tertullien rend compte de la croyance populaire en trois déesses, Lucine, Diane et Junon, qui président à l'accouchement (fées ventrières), suivies des Fata scribunda (qui écrivent le sort du nouveau-né). Le thème des fées marraines est attesté à l'époque médiévale aux alentours de l'an mil, et le motif du repas des fées apparaît dans le Roman de Perceforest, où la belle Zélandine, se piquant avec sa quenouille, tombe dans un sommeil profond (cf. l'article de N. Chardonnens). Le motif du repas des fées sera exploité dans la littérature médiévale, en particulier dans les chansons de geste tardives. Du mythe à la littérature, le motif se transforme et fait même l'objet de parodies. De manière générale, les déesses ont perdu leurs attributs de Parques pour devenir des "fées" au sens plus moderne du terme: les antiques Parques voient leurs pouvoirs élargis, métamorphosés au gré de l'imagination des auteurs et de 
mythologie antique est adaptée et actualisée à partir de projets singuliers dans les œuvres littéraires, même si l'on observe souvent une transposition des déesses en fées, et l'inversion (ou dédoublement) des rôles et des personnages (masculin et féminin, féerique et diabolique). A l'instar des figures antiques, la fée est ambivalente: détentrice d'un savoir occulte, elle est figure maternelle (Mélusine) ou objet de séduction dangereuse (Morgane). Mais elle pose toujours la question du statut de la femme et de la féerie comme royaume féminin associé à la sexualité et l'amour libre, opposé à la réalité du monde féodal masculin et patriarcal. La question du féminin est indissociable de la naissance du conte de fées comme genre littéraire chez Marie-Catherine d'Aulnoy et Charles Perrault, où le merveilleux sert à commenter la condition des femmes sous l'Ancien Régime ${ }^{11}$. D'ailleurs, l'écrivain féministe Angela Carter ne s'y trompera pas en leur rendant hommage dans ses réécritures. Au-delà des thèmes communs abordés dans cette partie, les contributions témoignent de la singularité de chaque nouvelle réalisation du conte et de la poétique complexe qui s'élabore à partir de stratégies intertextuelles ingénieuses et subtiles. L'analyse comparative démontre ainsi que les figures des fées marraines, malgré la proximité de La Belle au bois dormant et de Dornröschen, diffèrent significativement, les Grimm allant puiser leurs weise Frauen dans un fonds mythologique susceptible de créer un (pseudo-)folklore germanique.

L'histoire de Méléagre rassemble tous les éléments propres au monde gréco-romain avec des variantes ovidiennes que Jacqueline Fabre-Serris analyse dans «L'histoire de Méléagre vue par Ovide ou de quoi le tison des Parques est-il l'emblème?» La durée de la vie du héros n’a pas été impartie à la naissance par les Parques; celles-ci ont délégué ce pouvoir à sa mère en liant l'existence de Méléagre au tison enlevé du feu. Le traitement littéraire du récit consacre l'importance des femmes dans la destinée du héros. Ovide construit un récit narratif sous la forme d'épisodes

l'influence d'autres genres et traditions (B. Ribémont, "Laurence Harf-Lancner, Le Monde des fées dans l'Occident médiéval»).

II. La poétique particulière du conte de fées et ses enjeux sociopolitiques ont fait l'objet de plusieurs études ces dernières années. On citera L. C. Seifert, Fairy Tales, Sexuality and Gender in France (1690-1715); J. Mainil, Madame d'Aulnoy et le rire des fées; J.-P. Sermain, Métafictions (1670-1730) et Le Conte de fées du classicisme aux Lumières. On lira aussi avec profit la revue Féeries, et son homologue anglophone Marvels \& Tales: Journal of Fairy-Tale Studies. 
successifs où chacune a sa part: Diane, qui envoie le sanglier de Calydon comme épreuve de courage, Atalante, dont Méléagre tombe amoureux, sa mère Althée qui prend la décision fatale de remettre le tison dans le feu, ses sœurs qui le pleurent. Le tison magique symbolise ainsi le pouvoir secret des femmes sur le héros. Il est aussi le signe d'une double naissance: Althée lui a donné la vie une première fois en accouchant, et une deuxième fois en retirant le tison du feu. L'objet devient l'emblème original de ces liens invisibles et tout-puissants que chaque homme a, de sa naissance à sa mort, avec les femmes de sa famille.

Jean-Claude Mühlethaler poursuit avec la question du féminin et de l'héritage antique dans "Translittérations féeriques au Moyen Age: de Mélior à Mélusine, entre histoire et fiction ». L'article dénoue l'écheveau des sources mythologiques, littéraires et folkloriques dans plusieurs récits médiévaux où figurent des fées. Il montre comment Partenopeu de Blois se rattache au récit d'Apulée et au folklore celtique, reflétant à la fois des croyances populaires et des contraintes liées au genre du roman courtois. Au-delà du merveilleux symbolisé par la fée, ces récits soulèvent la question du statut de la femme au Moyen Age, de même que la place de l'amour au sein de la société féodale. Plus tard, Jean d'Arras utilisera la légende de la fée poitevine dans sa Mélusine à des fins plus directement idéologiques et politiques. Liée au lignage des Lusignan, la fée y est érotisée en épouse et mère. Elle connaîtra un vif succès, comme en témoignent les nombreuses éditions de la Bibliothèque Bleue.

L'article de Noémie Chardonnens illustre lui aussi la persistance des questionnements poétiques et politiques qui se nouent autour des figures des fées marraines à cette époque. "D'un imaginaire à l'autre: la belle endormie du Roman de Perceforest et son fils" traite du roman arthurien en prose de la fin du Moyen Age qui propose l'une des premières versions littéraires de l'histoire de La Belle au bois dormant. Plusieurs figures surnaturelles interviennent dans les scènes de naissance de l'héroïne et de son fils, l'article montrant quel rôle elles sont amenées à jouer dans la destinée des personnages. Bien que l'imaginaire de la naissance et de la petite enfance reflète les croyances populaires de cette époque, il participe essentiellement d'une logique romanesque issue des romans arthuriens antérieurs.

Dans la continuité de ses travaux sur les contes de Perrault, Ute Heidmann s'éloigne résolument de la piste folklorique pour relire $L a$ 
Belle au bois dormant à la lumière des textes antiques ${ }^{12}$. Elle montre dans "Tisserandes fatales (Apulée) et Fées de Cour (Perrault). Le sort difficile d'une Belle "née pour être couronnée" " que loin d'être un conte simple et naïf puisé dans un présumé folklore, le conte de Perrault s'élabore à partir d'un dialogue complexe avec la fabella d'Apulée et d'autres œuvres latines, italiennes et françaises. Là encore, La Belle au bois dormant est l'occasion de débattre du statut des femmes sous l'Ancien Régime, et notamment du sort des jeunes femmes de l'aristocratie et de la haute bourgeoisie française en âge de se marier. Parce qu'il occupe la première place dans le recueil, La Belle au bois dormant devient un conte programmatique où le lecteur peut trouver la clé de la poétique et de la politique du genre; celle-ci est à découvrir dans les figures des fées que l'auteur invite à décrypter selon la formule employée dans la préface dédicatoire au volume.

Sylvie Ballestra-Puech approfondit quant à elle un aspect de son ouvrage de référence sur les Parques. Elle s'intéresse ici à leur réception dans l'œuvre de Marie-Catherine d'Aulnoy, écrivaine contemporaine de Perrault et en quelque sorte marraine du genre, puisqu'elle lui a donné son nom de conte de fées, ou conte des fées. "Du fil des Parques au fil des fées: la fabrique du conte dans Serpentin vert de Madame d'Aulnoy" dénoue les liens complexes que d'Aulnoy tisse avec l'héritage littéraire antique afin d'élaborer une réflexion sur le genre, la condition féminine, et le rapport amoureux. L'article s'attache à l'épreuve du filage imposée à l'hérö̈ne de Serpentin vert par la fée Magotine (qui assume le rôle de Vénus dans le conte d'Amour et Psyché) dans un jeu intertextuel subtil qui fait de ce conte un manifeste esthétique. Ce motif se justifie d'abord en tant que réminiscence du texte d'Apulée et de sa réécriture par La Fontaine dans Les Amours de Psyché et Cupidon. En suggérant que son héroïne doit filer son propre destin et celui de son amant, d'Aulnoy condense en une image son interprétation de Psyché comme «belle farouche", tout en exploitant les résonances mythologiques et les riches potentialités symboliques du fil. La figure d'Arachné s'associe ainsi à celle de la Parque, pour citer Ballestra-Puech, pour symboliser le passage du fil du destin à celui de l'écriture.

I2. U. Heidmann, J.-M. Adam, Textualité et intertextualité des contes. Les travaux conjoints de Heidmann et Adam démontrent l'apport des approches interdisciplinaires pour l'étude des contes littéraires. 
A son tour, Magali Monnier se penche sur l'héritage de Madame d'Aulnoy dans la littérature contemporaine anglaise. Dans "Naissance et renaissance du conte de fées: de Marie-Catherine d'Aulnoy à Angela Carter", elle propose une lecture croisée de la dimension métapoétique déployée à partir de la métaphore filée du palais de féerie dans Gracieuse et Percinet et La Chatte blanche, et de la demeure de la Bête dans The Courtship of $M r$ Lyon. Cette réécriture qui entremêle des références aux contes de Beaumont et d'Aulnoy vise à renouveler le genre du conte de fées à partir d'une perspective contemporaine et féministe. Monnier montre comment le dialogue intertextuel qui se noue entre ces textes lie la thématique à des considérations esthétiques et politiques sur la dimension "genrée» du conte, qui aborde la condition des femmes à leur époque respective ${ }^{13}$.

Pour sa part, Cyrille François propose une analyse textuelle contrastive des fées de Perrault et de leurs homologues germaniques chez les Grimm, dans «Fées et weise Frauen: les faiseuses de dons chez Perrault et les Grimm, du merveilleux rationalisé au merveilleux naturalisé». Il montre que ces personnages du conte français et du Märchen allemand ne présentent que des ressemblances superficielles: les fées et les weise Frauen ne jouent pas le même rôle dans l'intrigue, et elles agissent selon des logiques propres. Si le conte de Perrault motive les actions des fées et en fait des êtres réfléchis, le Märchen des Grimm représente les weise Frauen comme des figures énigmatiques appartenant à un univers mystérieux qui puise dans un fond mythologique. Le traitement de ces personnages féminins emblématiques reflète une volonté de rationaliser le merveilleux chez Perrault qui annonce les Lumières, contrairement aux Grimm dont l'œuvre plus tardive s'inscrit dans le contexte de la construction des identités nationales européennes.

Larticle de Donald Haase qui clôt cette deuxième partie établit le lien entre la filiation littéraire et la tradition orale et populaire du conte dans "Kiss and Tell: Orality, Narrative, and the Power of Words in "Sleeping Beauty" " ${ }^{14}$. Il s'attache à la dimension métafictionnelle du conte, mais à partir de la tension entre parole et écriture qui confère à l'histoire de La Belle au bois dormant une dimension autoréflexive significative, où

I3. Voir E. Wanning Harries, Twice Upon A Time.

I4. Voir aussi dans le domaine francophone A. Defrance, J.-F. Perrin (éds), Le conte en ses paroles. 
l'on peut lire dans le motif du baiser une métaphore de la réception du conte. L'auteur examine comment l'activité et la manière de raconter une histoire sont représentées non seulement dans les versions classiques de Perrault et de Grimm, mais aussi dans le Roman de Perceforest qui les inspire et, au $\mathrm{XX}^{\mathrm{e}}$ siècle, dans le célèbre dessin animé de Walt Disney. Cette contribution annonce la dernière partie du volume en ouvrant la perspective sur des modes de transmission non exclusivement textuels et littéraires pour s'intéresser à la transmission culturelle du conte jusqu'à aujourd'hui.

Transmission culturelle et époque contemporaine: paroles, textes, images

La troisième partie rassemble des contributions qui documentent la réception littéraire et visuelle, mais aussi plus largement culturelle du conte de La Belle au bois dormant. Qu'elles s'inspirent directement des textes de Perrault et de Grimm ou de son stéréotype international ${ }^{15}$, les réécritures et adaptations contemporaines témoignent de la continuité des questions liées à la représentation et au rôle des femmes dans le conte, ainsi qu'à des considérations sur la politique du genre et ses implications sociales et idéologiques. L'érotisation de la belle endormie ou de la fée fatale fait notamment l'objet de critiques féministes au $\mathrm{XX}^{\mathrm{e}}$ siècle. On observe aussi des retours à l'héritage "nocturne" des Parques antiques et à la face sombre du conte familier en réaction à la "disneyfication » du genre, de même qu'un renouveau d'intérêt pour l'oralité et les "mauvais» genres (fantastique, pornographie, BD, etc.). Au XIX' siècle, l'œuvre de Gustave Doré est indissociable de la réception moderne des contes de Perrault, ainsi que les productions fin-de-siècle qui exploitent les "perversions du merveilleux", pour reprendre l'heureuse formule de Jean de Palacio ${ }^{16}$. Cette réception décadente est soumise à la critique d'Angela Carter dans The Bloody Chamber (1979), qui témoigne aussi de la présence de La Belle au bois dormant dans la culture populaire. De nombreuses œuvres d'art, romans et films récents ouvrent à nouveau la question de la représentation de la belle endormie pour aborder des thèmes contemporains comme le vieillissement et la sexualité, mais aussi

15. C. Dollerup, Tales and Translation.

I6. J. de Palacio, Les Perversions du merveilleux. 
et toujours le mystère de la beauté, du rêve et de l'inspiration. Enfin, deux réécritures du conte pour la jeunesse développent les capacités du conte à traiter de problématiques liées à l'histoire violente du $\mathrm{XX}^{\mathrm{e}}$ siècle afin de témoigner d'un vécu indicible, et transmettre une expérience, des valeurs, voire une «morale utile» (pour citer Perrault) destinée aux enfants et aux jeunes adultes.

Le thème du destin fatal hante certaines œuvres tardives de Gustave Doré, plus connu pour ses illustrations des contes de Perrault et autres classiques de la littérature occidentale. Comme le montre Philippe Kaenel dans «Féerique et macabre: l'art de Gustave Doré», la conception du merveilleux de l'artiste est empreinte d'une dimension funeste qui offre un éclairage inédit sur son œuvre. Si le célèbre illustrateur contribue à modeler durablement l'imaginaire lié au conte, on sait moins que ses créations souvent macabres et sanguinaires font figurer le motif de la Parque de manière récurrente. Ainsi, dans son illustration du poème de Coleridge, The Rime of the Ancient Mariner, la Mort fait référence à la figure d'Atropos, tandis que le groupe sculpté de La Parque et l'Amour, présenté au Salon de 1877, montre l'Amour soumis à la Mort personnifiée elle aussi par Atropos. Ses illustrations du poème d'Edgard Poe "The Raven» renouent également avec l'héritage antique en associant le féerique et le fatidique.

Les fées fatidiques, amantes capricieuses et muses inspiratrices figurent en bonne place dans la littérature fin-de-siècle inspirée des contes. Michel Viegnes, dans «La force au féminin dans le conte merveilleux fin-de-siècle", documente un paradoxe bien connu de la fin-de-siècle européenne: en réaction au positivisme, matérialisme et naturalisme triomphants, des écrivains et des artistes se tournent vers des mondes imaginaires souvent marqués par l'esthétique décadente du pervers et du morbide. Le genre du conte merveilleux est remis à l'honneur par des poètes tels que Théodore de Banville, Catulle Mendès, Henri de Régnier, et des prosateurs comme Marcel Schwob. Qu'elles soient fée, femme-enfant ou princesse faussement ingénue, les figures féminines sont fondamentalement ambiguës: détentrices d'un savoir ou de facultés surnaturelles qui les rendent redoutables, les très jeunes femmes des contes fin-de-siècle sont aussi des réminiscences d'un monde révolu et condamné par la modernité, et peut-être même de l'art lui-même, comme le suggère la belle endormie de "La Belle au bois rêvant" de Catulle Mendès. 
Témoignant elle aussi de la part sombre du conte, la Belle d'Angela Carter est fille rebelle de Nosferatu. Martine Hennard Dutheil de la Rochère, dans "Conjuring the Curse of Repetition or Sleeping Beauty Revamped: Angela Carter's Vampirella and The Lady of the House of Love», montre comment Angela Carter revisite La Belle au bois dormant dans deux histoires de vampire, la pièce radiophonique Vampirella et sa réécriture en prose, The Lady of the House of Love. Elle montre comment la vamp devient figure de l'auteur, qui vampirise la culture européenne et transfuse les vieilles histoires dans de nouvelles formes, genres et médias afin de leur donner une nouvelle vie, tout en offrant un éclairage inédit sur le conte de Perrault. Ce travail de relecture du conte à rebours des idées reçues se double de sa transposition dans la culture populaire, où la femme fatale, inspirée par le fanzine et la bande dessinée, échappe à son destin.

A la suite des travaux féministes sur le regard masculin (the male gaze) et l'objectification de la femme dans l'art occidental, Elizabeth Wanning Harries s'interroge sur la politique de genre à l'œuvre dans la réactivation du topos de la "belle endormie" dans certaines adaptations récentes du conte. "Old Men and Comatose Virgins: Nobel Prize Winners rewrite "Sleeping Beauty" " examine la façon dont ces écrivains réactivent une fantasmatique masculine qui érotise le spectacle de très jeunes femmes endormies. A la différence des contes classiques, celles-ci ne se réveillent pas chez Kawabata et García Márquez. En revanche, les belles endormies donnent une nouvelle vie aux vieux messieurs qui les contemplent et les caressent alors qu'elles sont plongées dans un profond sommeil. Dans ces romans, les jeunes filles sont représentées comme des poupées désirables mais sans personnalité ni individualité. Elles sont manipulées par des femmes plus âgées, patronnes de maisons closes qui remplacent les fées traditionnelles et déterminent leur sinistre destin. Or, ces romans déplacent l'attention de la jeune fille endormie sur les vieillards euxmêmes, leur peur de vieillir, et leur obsession perverse et mortifère pour les jeunes filles endormies. Wanning Harries élargit le propos en mettant en évidence une véritable fascination pour la figure de la belle endormie dans la culture visuelle et cinématographique contemporaine.

La Belle au bois dormant figure en bonne place parmi les contes classiques destinés à la jeunesse. "La disparition des fées dans El verdadero final de la Bella Durmiente d'Ana María Matute» de Sylvie Ravussin, s'attache à la façon dont l'écrivaine contemporaine espagnole Ana 
María Matute réécrit et adapte le conte de Perrault pour les enfants. Elle montre comment le conte français est reconfiguré en un cuento espagnol qui recourt notamment au fantastique et à la disparition du merveilleux, symbolisé par le personnage de la fée, pour signaler la perte de l'enfance et les sombres réalités de l'Espagne sous le régime franquiste. $E l$ verdadero final de la Bella Durmiente s'inscrit ainsi dans un projet poétique élaboré sur une longue période, dans le prolongement du roman autobiographique de Matute, Primera memoria.

La dimension (auto)biographique et mémorielle est elle aussi au cœur du roman que Jane Yolen destine à de jeunes adultes. Martine Hennard Dutheil de la Rochère et Géraldine Viret, dans «Sleeping Beauty in Chelmno : Jane Yolen's Briar Rose or Breaking the Spell of Silence» s'intéressent à la transposition des contes de Perrault et des Grimm dans le contexte de l'Holocauste. A partir des études sur le traumatisme (trauma studies), l'article montre comment le conte de fées est mobilisé dans le roman de Yolen pour communiquer une expérience indicible. Il s'attache en particulier au rôle de Becca, co-témoin placé au cœur du dispositif narratif, chargée de transmettre à travers le conte familier l'histoire de sa grand-mère et à travers elle une mémoire collective menacée par l'oubli. Liant les aspects poétique, didactique, politique et éthique du genre, le roman aborde à travers le conte la question épineuse de la représentation de l'Holocauste et de la «fictionalisation» de l'histoire qui accompagne la disparition des témoins directs.

Des divinités antiques aux fées (post)modernes, le volume témoigne de la richesse et de la diversité des réalisations et réinterprétations des figures féminines associées à l'imaginaire de la naissance, au croisement des traditions mythopoétiques anciennes et des stratégies de réécriture et d'adaptation modernes: outre les rapports complexes entre culte, mythe et conte, parole et écriture, texte et image, il aborde le rôle des femmes, divines et humaines, dont le verbe magique a le pouvoir de déterminer le destin. Dans la tradition littéraire, les fées sont souvent les messagères de la dimension critique et politique du conte, inséparable de sa poétique; mais comme dans le monde antique, le merveilleux est marqué par l'ambivalence et sa face sombre et inquiétante transparaît dans l'œuvre de nombreux artistes et écrivains. On observe enfin que le «conte» s'élabore dans une dynamique de reprise et d'actualisation, de transposition et de transformation dans des genres, formes et médias divers. Il est à la fois 
porteur d'une mémoire collective, transmise de génération en génération, et création singulière.

Martine Hennard Dutheil de la Rochère Université de Lausanne Véronique DASEN Université de Fribourg 


\section{BIBLIOGRAPHIE}

Anderson, Graham, Fairytale in the Ancient World, London, Routledge, 2000.

Ballestra-Puech, Sylvie, Les Parques. Essai sur les figures du destin dans la littérature occidentale, Toulouse, Editions universitaires du Sud, 1999.

Bottigheimer, Ruth B., Fairy Tales: A New History, New York, State University of New York Press, 2009.

Crane, Walter, The Bluebeard Picture Book, London, George Routledge and Sons, 1875.

Defrance, Anne, Perrin, Jean-François (éds), Le conte en ses paroles: La figuration de l'oralité dans le conte merveilleux du Classicisme aux Lumières, Paris, Desjonquères, 2007.

Dollerup, Cay, Tales and Translation: The Grimm Tales from Pan-Germanic Narratives to Shared International Fairytales, Amsterdam/Philadelphie, Benjamins Translation Library, V. 30, 1999.

FARAL, Edmond, Recherches sur les sources latines des contes et romans courtois du Moyen Age, Genève, Slatkine, 1983.

Hanse, Donald (ed.), Fairy Tales and Feminism: New Approaches, Detroit, Wayne State University Press, 2004.

Harf-Lancner, Laurence, Les fées au Moyen Age: Morgane et Mélusine. La naissance des fées, Paris, Champion, 1991 [1984].

—, Les mondes des fées dans l'occident médiéval, Paris, Hachette, 2003.

Harries, Elisabeth Wanning, Twice Upon A Time: Women Writers and the History of the Fairy Tale, Princeton, Princeton University Press, 2001.

Heidmann, Ute, Adam, Jean-Michel, Textualité et intertextualité des contes. Perrault, Apulée, La Fontaine, Lhéritier..., Paris, Classiques Garnier, 2010. 
Horst, P. C. van der, "Fatum, Tria Fata; Parca, Tres Parcae", Mnemosyne, 11 (1943), p. 217-227 (http://www.jstor.org/ stable/4427045).

Mainil, Jean, Madame d'Aulnoy et le rire des fées. Essai sur la subversion féerique et le merveilleux comique sous l'Ancien Régime, Paris, Kimé, 2001.

Palacio, Jean de, Les Perversions du merveilleux, Paris, Séguier, 1993.

Ribémont, Bernard, "Laurence Harf-Lancner, Le Monde des fées dans l'Occident médiéval", Cahiers de recherches médiévales et humanistes, 2003 (http://crm.revues.org//229).

Seifert, Lewis C., Fairy Tales, Sexuality and Gender in France (16901715). Nostalgic Utopias, Cambridge, Cambridge University Press, 1996.

Sermain, Jean-Paul, Métafictions (1670-1730). La réflexivité dans la littérature d'imagination, Paris, Honoré Champion, 2002.

—, Le Conte de fées du classicisme aux Lumières, Paris, Desjonquères, 2005.

Sorlin, Irène, "Striges et Geloudes. Histoire d'une croyance et d'une tradition", Travaux et Mémoires. Centre de recherche d'histoire et de civilisation Byzantine, 11 (1991), p. 411-436.

Velay-Vallantin, Catherine, L'histoire des contes. Nouvelles études historiques, Paris, Fayard, 1992.

Warner, Marina, From the Beast to the Blonde. On Fairy Tales and their Tellers, New York, Chatto \& Windus, 1994.

—, "After Rapunzel», Marvels \& Tales, 24/2 (2010), p. 329-335.

Zipes, Jack (éd.), The Oxford Companion to the Fairy Tale, Oxford, Oxford University Press, 2000.

\section{Crédit iconographique}

Crane, Walter, The Bluebeard Picture Book, London, George Routledge and Sons, 1875. (http://www.archive.org/stream/ sleepingbeautypi $00 \mathrm{cran} \#$ page/n $7 /$ mode/2up) 